\title{
Mucosal Immunity and the Microbiome
}

\author{
Andrew S. Neish ${ }^{1}$ \\ ${ }^{1}$ Department of Pathology, Emory University School of Medicine, Atlanta, Georgia
}

\begin{abstract}
By definition, the mucosal immune system is responsible for interfacing with the outside world, specifically responding to external threats, of which pathogenic microbes represent a primary challenge. However, it has become apparent that the human host possesses a numerically vast and taxonomically diverse resident microbiota, predominantly in the gut, and also in the airway, genitourinary tract, and skin. The microbiota is generally considered symbiotic, and has been implicated in the regulation of cellular growth, restitution after injury, maintenance of barrier function, and importantly, in the induction, development, and modulation of immune responses. The mucosal immune system uses diverse mechanisms that protect the
\end{abstract}

host from overt pathogens, but necessarily has coevolved to monitor, nurture, and exploit the normal microbiota. As a whole, mucosal immunity encompasses adaptive immune regulation that can involve systemic processes, local tissue-based innate and inflammatory events, intrinsic defenses, and highly conserved cell autonomous cytoprotective responses. Interestingly, specific taxa within the normal microbiota have been implicated in roles shaping specific adaptive, innate, and cell autonomous responses. Taken together, the normal microbiota exerts profound effects on the mucosal immune system, and likely plays key roles in human physiology and disease.

Keywords: signaling; inflammation; microbiota; probiotics; reactive oxygen

(Received in original form June 12, 2013; accepted in final form October 1, 2013)

Correspondence and requests for reprints should be addressed to Andrew S. Neish, M.D., 105-L Whitehead Building, Department of Pathology and Laboratory Medicine, Emory University School of Medicine, 615 Michaels Street, Atlanta, GA, 30322. E-mail: aneish@emory.edu

Ann Am Thorac Soc Vol 11, Supplement 1, pp S28-S32, Jan 2014

Copyright (C) 2014 by the American Thoracic Society

DOI: 10.1513/AnnalsATS.201306-161MG

Internet address: www.atsjournals.org

\section{Eukaryotic/Prokaryotic Interactions at the Mucosal Surface}

Commensal host-microbe interactions have coevolved over millennia in many animals, with the human luminal ecosystems representing a highly medically relevant example (1). The vast majority of these microbes comprise about 500 genera of bacteria, broadly grouped into 2 taxonomic divisions: the Bacteroidetes and Firmicutes (2). An accurate accounting of the microbiota is not practical by conventional microbiological techniques; however, highthroughput sequencing and molecular taxonomic methodologies have greatly increased our understanding of the population composition, dynamics, and ecology of the microbiota (3).

This complex microbial ecosystem is separated from the host interior by only a single layer of epithelial cells present in the gastrointestinal tract (site of by far the greatest microbial populations), airways, genitourinary tract, and skin. Epithelial cells, by definition, act as interfaces between the host and the environment, and are equipped with apical surface specializations (microvilli, cilia, mucus production, intercellular junctions) to permit physiological function while contacting the microbiota. In addition, studies with germfree mice have revealed that the microbiota is not functionally insulated from the mucosa, but in contrast, resident bacteria can fundamentally influence epithelial metabolism, proliferation and survival, and barrier function (4). For example, the small intestinal villi of the germ-free gut are elongated, whereas crypts are atrophic, and show a slower turnover of the epithelial cells and defective angiogenesis. Such mice monocolonized with single gut-symbiont species (Bacteroides thetaiotaomicron) exhibit robust host transcriptional responses, indicating that host recognition of the microbiota occurs.

Intestinal bacteria thrive in a stable, nutrient-rich environment but also serve beneficial functions to the host including energy salvage of otherwise indigestible complex carbohydrates, vitamin and micronutrient syntheses, competitive exclusion of pathogenic microorganism, and importantly, stimulation of immune development (5). Thus there is a dynamic interaction between the microbiota and the mucosa, resulting in for the most part a mutually beneficial relationship. However, in other cases, a "dysbiotic" microbiota may be sufficient to provoke intestinal inflammation, such as that seen in inflammatory bowel disease, and there is much current interest in quantitative and/or qualitative abnormalities of the microbiota that may be associated with systemic immune, allergic, metabolic, and infectious disorders (6). In the airway, there 
is increasing recognition and interest in the effects of resident microbiota in respiratory homeostasis and disease processes $(7,8)$. Quantitative alterations the microbiota have been described in chronic obstructive pulmonary disease, asthma, and cystic fibrosis (9-11). Finally, there is also increasing interest in potential therapeutic benefits of supplementing the microbiota with exogenous viable bacteria. This approach, termed probiotics, has been reported to dampen inflammation, stimulate local and systemic immune development, and improve reparative responses in vitro and has shown promise as therapy in several inflammatory and immune disorders (12). Thus, there is increasing and compelling evidence that the human microbiota beneficially affects systemic homeostasis and thus health.

\section{The Microbiota and Systemic Effects: Adaptive Immune Stimulation}

Especially in the context of the gut, the host has developed highly conserved interrelated systems to protect itself from pathogenic attack, but to allow beneficial microbes-the microbiota-to thrive (Figure 1). The mucosal arm of the adaptive immune system provides humoral and cell-mediated immunity against ingested (and inhaled) antigens and luminal organisms, and is most fully developed in the small bowel. A large amount of IgA is secreted across mucosal surfaces. Effector lymphocytes are diffusely distributed in the lamina propria, in isolated lymphoid follicles, or organized into discrete structures termed Peyer's patches, which are essentially mucosal lymph nodes overlaid with a specialized epithelial cell type, the $M$ cell, which possesses the endocytic machinery for uptake of particulate antigens from the gut lumen. Members of the microbiota, along with nonviable particulate antigens and all-too-viable pathogens, are continually being sampled by the $M$ cells and perhaps other portals for processing by local dendritic cells or macrophages and subsequent education and activation of effector B and T cells (13).

The microbiota is clearly involved in the anatomic and functional development of mucosal adaptive immunity (14). Peyer's patches are grossly hypoplastic and IgA responses are reduced in germ-free animals.
It is also known that germ-free animals have reduced total $\mathrm{CD} 4^{+} \mathrm{T}$-cell populations and an inappropriate balance of helper T-cell subsets, which can be moderated within weeks on colonization with a representative member of the normal flora (Bacteroides fragilis) via dendritic cell recognition of a specific polysaccharide (polysaccharide A) component of $B$. fragilis. The intestinal lamina propria in healthy animals is a major location of a unique population of IL-17-producing $\mathrm{CD} 4{ }^{+} \mathrm{T}$ cells (Th17 cells), distinct from Th1 or Th2 cell lineages. It has been demonstrated that Th17-cell development is dependent on a single taxon, the segmented filamentous bacteria present in the murine microbiota and living in physical contact with the epithelia. In addition, Clostridia species have been implicated in development of mucosal T-regulatory cells. Collectively, these observations demonstrate a key role of the microbiota in the development and education of the mucosal adaptive immune system, and interestingly implicate specific members in these developmental

functions (15).

\section{The Microbiota and Tissue Effects: Innate Immune Regulation}

Epithelial and mucosal immune cells are capable of inducing local inflammatory responses. Transmembrane and intracytoplasmic receptors, such as the now well-studied Toll-like receptors (TLRs) and related Nod proteins, are designated "pattern recognition receptors" or PRRs. PRRs recognize and bind to "microbeassociated molecular patterns," conserved structural motifs present on the surface of a wide range of microbes. For example, TLR4 recognizes lipopolysaccharide and TLR2 binds specific peptidoglycans-both components of bacterial cell walls. The now well-known association of Crohn's disease with mutant forms of Nod2 clearly underscores the importance of PRR monitoring in health (16).

PRRs and their downstream signaling pathways, such as the mitogen-activated protein kinase and NF- $\mathrm{KB}$ systems, have an ancient lineage, exhibiting impressive structural and functional homology even at the level of invertebrates and plants. These systems represent entwined cytoplasmic information relays, which when activated employ rapid posttranslational events (covalent protein modifications and regulated protein degradation) to transduce PRR binding into well-defined inflammatory and apoptotic tissue responses designed to eliminate pathogenic threats (17). For example, inflammatory signaling is necessary for chemotaxis and recruitment of inflammatory cells required for the phagocytosis of invading bacteria and subsequent microbicidal elimination via the respiratory burst. Phagocytosis is also required for the clearing of cellular debris postinjury. Apoptotic networks allow infected or otherwise compromised cells to be selectively eliminated without damage at the tissue level. In addition, it is also well understood that innate immune signaling in also required for the induction and maintenance of adaptive responses.

Although it is obvious that the host must defend against threats posed by bacterial pathogens, the benefits conferred by the microbiota require that immune and inflammatory systems not eliminate them entirely. The epithelia can suppress TLR signaling or reduce TLR expression to moderate immunoinflammatory signaling (18). In addition, individual members of the microbiota are able to actively modulate signaling intensity. A variety of reports have described commensals-many employed as probiotics-that are able to suppress eukaryotic inflammatory signaling pathways such as NF- $\mathrm{kB}$ and block inflammatory effector functions (19).

Several laboratories have demonstrated that intestinal bacteria are able to influence inflammatory pathways, and likely other cellular regulatory processes, by manipulating the ubiquitin system. Ubiquitination is a covalent modification increasingly recognized to play a regulatory role in a wide spectrum of biochemical events, generally by targeting modified proteins for controlled degradation via the proteasome organelle. An example of a signaling module regulated by ubiquitination is the inhibitory component of the NF- $\kappa \mathrm{B}$ pathway, IкB, and there are numerous examples of pathogens that use preformed effector proteins to influence I $\kappa$ B ubiquitination and thus innate immunity (20). Members of the microbiota interacting with epithelial cells in vitro are capable of blocking IкB ubiquitination and thus NF-кB activation by interference with the function of the IкB ubiquitination ligase, $\mathrm{SCF}^{\beta \mathrm{TrCP}}$ (Skp1, Cdc53/Cullin, $F$ box receptor) 
$(21,22)$. This enzymatic complex is activated by a second covalent modification, neddylation, on the regulatory subunit of the complex, cullin-1. Neddylation is the covalent modification of the SCF ubiquitin ligase by the ubiquitin-like protein Nedd8, and is a central regulatory event in cellular processes that are controlled by protein degradation, including NF- $\kappa \mathrm{B}$ and $\beta$-catenin. Neddylation occurs by an enzymatic series analogous to the ubiquitination reaction, specifically catalyzed by a Nedd8 ligase called Ubc12. We have shown that contact of commensal bacteria with epithelia in vitro and in vivo resulted in the rapid and reversible loss of the Nedd 8 modification, accounting for the loss of overall SCF ubiquitin ligase function and consequent blockade of NF- $\kappa$ B activation (22). Prompted by observations that other enzymes involved in modification of regulatory proteins by ubiquitin-like enzymes (the SUMOylation process) were controlled by transient oxidative inactivation, we investigated whether the neddylation reaction was influenced by oxidative signaling. We demonstrated that both endogenous reactive oxygen species (ROS; $\mathrm{H}_{2} \mathrm{O}_{2}$ ) and ROS generation by bacterial contact were able to transiently inactivate the Nedd8 ligase, Ubc12 (23). These results demonstrated that commensal bacteria directly modulate a critical control point of the ubiquitinproteasome system, the first example of a eukaryotic signaling pathway influenced via bacterially stimulated ROS, and furthermore provide a detailed molecular mechanism for bacterial suppression of the host inflammatory pathway.

Taken together, these observations demonstrate that although pathogen recognition is a key role of mucosal innate immunity, members of the microbiota can influence these processes, likely in the form of cross-talk leading to a "negotiated settlement" between the mucosa and the resident microbiota.

\section{The Microbiota and Cell Autonomous Effects: Cytoprotection, Growth, and Survival}

Although PRR-mediated signaling clearly has a central and dominant role in initiating cellular inflammation during infection, it is now also apparent that basal tonic PRRmediated signaling in response to the normal microbiota and their products is necessary for mucosal homeostasis. Murine mutants with defective PRR signaling are hypersensitive to a variety of intestinal and systemic insults and stressors, and supplementation of TLR ligands such as CpG DNA and flagellin can have cytoprotective effects (24). Regenerative responses to colonic injury are markedly attenuated in germ-free animals, indicating a discernable role of the microbiota in the stimulation of epithelial proliferation and response to injury, and restitution is reduced in MyD88 (a signaling intermediate required by multiple TLRs) null mice, reinforcing the notion that PRR-mediated signaling is necessary for trophic/restitutive effects (25). These and related observations with mice null in epithelial NF- $\kappa \mathrm{B}$ pathway components support the hypothesis that a constitutive degree of PRR signaling is necessary for normal gut homeostasis, presumably because of the tonic up-regulation of cytoprotective genes (gene products with antiapoptotic and chaperone/stress response) (26).

As mentioned, a cardinal feature of the acute inflammatory responses is phagocyte ROS production, which occurs in response to stimulation of formylated peptide receptors by prokaryotic peptides modified by a characteristic N-terminal formyl group. Formylated peptide receptor (FPR) stimulation occurs with both pathogenic and symbiotic bacteria. Importantly, immunohistochemical staining has shown the FPRs are expressed not solely on phagocytes, but also on the apical surface of the intestinal and airway epithelia, prompting interest that this and related epithelial receptors may mediate physiological responses in mucosal tissues (27). Phagocytes generate ROS via a well-studied enzymatic apparatus. The neutrophil NADPH oxidase, Nox2 (formerly gp $120^{\text {phox }}$ ), is a constitutively inactive multisubunit complex composed of a membrane-bound dimer of $\mathrm{p} 22^{\text {phox }}$ and gp91 ${ }^{\text {phox }}$ (28). The in vivo role of this enzyme in host defense is vividly illustrated by the fact that the genetic absence of Nox2 function results in chronic granulomatous disease, a condition in which phagocytes fail to induce ROS and patients are predisposed to recurrent pyogenic infections. In addition, it is now apparent that the ROS-generating enzymes activated by FPRs in neutrophils (Nox2) have functional paralogous enzymatic complexes in nonphagocytic cells (28). Indeed, a family of NADPH oxidase enzymes, the Nox and Duox enzymes, is

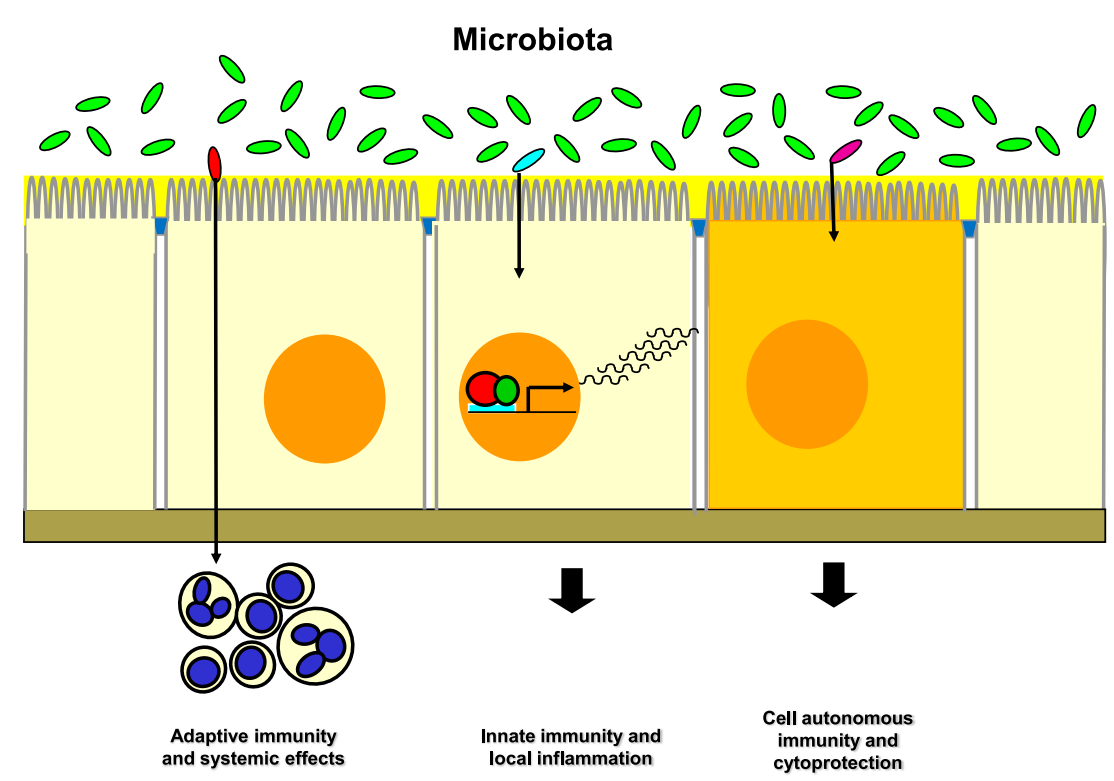

Figure 1. Effect of the microbiota on mucosal immunity. Shown is a schematic of the epithelial interface with the microbiota. Distinct taxa of the microbiota, depicted as various colors, can influence processes affecting adaptive, innate, and cell autonomous immunity. Commensal bacteria can influence T-cell differentiation at sites distant from the mucosa, modulate epithelial innate signaling pathways, and induce cytoprotective states. 
seen in many nonphagocytic tissues, with two, Nox1 and Duox2, strongly expressed in epithelial tissues. In general, the nonphagocytic NADPH oxidases exhibit similar, but not identical, organization to the phagocyte enzyme.

We have shown that several species of normal human gut bacteria can induce rapid, "deliberate" generation of ROS within epithelial cells (23), and this ROS production has significant signaling effects on innate immunity, proliferation, and epithelial movement and restitution $(19,29)$. Indeed, data in invertebrates suggest that ROS generation for signaling and microbiocidal functions in the gut epithelia may represent the ancestral form of response to bacteria (30). As mentioned, Nox enzymes play a central role in ROS generation in phagocytes; using Noxl and Nox2 null mice, we have demonstrated that rapid commensal-dependent ROS generation is abolished in Nox1 and FPR1 null animals but not in Nox2 null animals or mice with defective TLR signaling, indicating that commensal-mediated ROS generation occurs in the epithelial compartment. Furthermore, such mice, unable to induce epithelial ROS, do not manifest the proproliferative and prorestitutive effects of the microbiota.
In addition, we have shown that commensal-mediated ROS generation is a potent stimulus of the Nrf2/ARE pathway. The Nrf2 pathway is a well-studied cytoprotective pathway (31) that responds to both physiologically generated, endogenous ROS, and xenobiotic stresses. Redox stress allows for the stabilization of the Nrf2 transcription factor, with can enter the nucleus and stimulate activation of a battery of antioxidant and cytoprotective genes. Many workers have shown that Nrf2 null mice are hypersensitive to a wide range of injurious stimuli, including chemical- and radiationinduced mucosal injury. In normal mice, such stimuli can be ameliorated by colonization with probiotic bacteria; however, these beneficial effects are lost in Nrf2 null animals.

Interestingly, different strains of commensal bacteria can elicit marked differences in ROS level, redox signaling, and Nrf2 activation in contacted cells. We have found that the lactobacilli are especially potent stimulators of ROS production in cultured cells and in vivo, although all bacteria tested have some ability to alter the redox environment of the cell. Such high ROS-stimulating bacteria, such as the lactobacilli, may possess specific membrane components or even secreted factors that activate cellular ROS production.
Alternatively, high ROS-stimulating bacteria may simply possess enhanced adhesion or ability to penetrate mucin layers and gain more proximal access to cellular receptors such as the TLRs and FPRs.

Overall, these data indicate that the microbiota has profound effects on cellular homeostasis and underscores the importance of gut-prokaryotic interaction as a beneficial and necessary relationship.

\section{Conclusions}

In conclusion, mucosal immunity, like all immunity, functions to protect the host from exogenous, generally microbial threats. Paradoxically, we are becoming increasingly aware of the myriad benefits provided by our microbiota. Thus, it is hardly surprising that mutual coevolution has permitted physiologic-not pathologic-cross-talk to occur between the host and its prokaryotic residents. It is hoped that a fuller understanding of this relationship may advance our understanding of human health and disease.

Author disclosures are available with the text of this article at www.atsjournals.org.

\section{References}

1 Neish AS. Microbes in gastrointestinal health and disease. Gastroenterology 2009;136:65-80.

2 Bäckhed F, Ley RE, Sonnenburg JL, Peterson DA, Gordon JI. Hostbacterial mutualism in the human intestine. Science $2005 ; 307$ : 1915-1920.

3 Sommer F, Bäckhed F. The gut microbiota-masters of host development and physiology. Nat Rev Microbiol 2013;11:227-238.

4 Smith K, McCoy KD, Macpherson AJ. Use of axenic animals in studying the adaptation of mammals to their commensal intestinal microbiota. Semin Immunol 2007;19:59-69.

5 Marchesi J, Shanahan F. The normal intestinal microbiota. Curr Opin Infect Dis 2007;20:508-513.

6 Noverr MC, Huffnagle GB. Does the microbiota regulate immune responses outside the gut? Trends Microbiol 2004;12:562-568.

7 Dickson RP, Erb-Downward JR, Huffnagle GB. The role of the bacterial microbiome in lung disease. Expert Rev Respir Med 2013;7: 245-257.

8 Erb-Downward JR, Huffnagle GB, Martinez FJ. The microbiota in respiratory disease. Am J Respir Crit Care Med 2012;185: 1037-1038.

9 Han MK, Huang YJ, Lipuma JJ, Boushey HA, Boucher RC, Cookson WO, Curtis JL, Erb-Downward J, Lynch SV, Sethi S, et al. Significance of the microbiome in obstructive lung disease. Thorax 2012;67:456-463.

10 Huang YJ, Nelson CE, Brodie EL, Desantis TZ, Baek MS, Liu J, Woyke T, Allgaier M, Bristow J, Wiener-Kronish JP, et al. Airway microbiota and bronchial hyperresponsiveness in patients with suboptimally controlled asthma. J Allergy Clin Immunol 2011;127:372-381.e1-3.
11 Zhao J, Schloss PD, Kalikin LM, Carmody LA, Foster BK, Petrosino JF, Cavalcoli JD, VanDevanter DR, Murray S, Li JZ, et al. Decade-long bacterial community dynamics in cystic fibrosis airways. Proc Natl Acad Sci USA 2012;109:5809-5814.

12 Bienenstock J, Gibson G, Klaenhammer TR, Walker WA, Neish AS. New insights into probiotic mechanisms: a harvest from functional and metagenomic studies. Gut Microbes 2013;4:94-100.

13 Neish AS, Denning TL. Advances in understanding the interaction between the gut microbiota and adaptive mucosal immune responses. F1000 Biol Rep 2010;2:27.

14 Hooper LV, Littman DR, Macpherson AJ. Interactions between the microbiota and the immune system. Science 2012;336: 1268-1273.

15 Ivanov II, Honda K. Intestinal commensal microbes as immune modulators. Cell Host Microbe 2012;12:496-508.

16 Sartor RB. Microbial influences in inflammatory bowel diseases. Gastroenterology 2008;134:577-594.

17 Fukata M, Arditi M. The role of pattern recognition receptors in intestinal inflammation. Mucosal Immunol 2013;6:451-463.

18 Abreu MT, Fukata M, Arditi M. TLR signaling in the gut in health and disease. J Immunol 2005;174:4453-4460.

19 Swanson PA II, Kumar A, Samarin S, Vijay-Kumar M, Kundu K, Murthy $\mathrm{N}$, Hansen J, Nusrat A, Neish AS. Enteric commensal bacteria potentiate epithelial restitution via reactive oxygen speciesmediated inactivation of focal adhesion kinase phosphatases. Proc Natl Acad Sci USA 2011;108:8803-8808.

20 Rytkönen A, Holden DW. Bacterial interference of ubiquitination and deubiquitination. Cell Host Microbe 2007;1:13-22.

21 Neish AS, Gewirtz AT, Zeng H, Young AN, Hobert ME, Karmali V, Rao AS, Madara JL. Prokaryotic regulation of epithelial responses by inhibition of ІкB- $\alpha$ ubiquitination. Science 2000;289:1560-1563. 
22 Collier-Hyams LS, Sloane V, Batten BC, Neish AS. Cutting edge: bacterial modulation of epithelial signaling via changes in neddylation of cullin-1. J Immunol 2005;175:4194-4198.

23 Kumar A, Wu H, Collier-Hyams LS, Hansen JM, Li T, Yamoah K, Pan ZQ, Jones DP, Neish AS. Commensal bacteria modulate cullin-dependent signaling via generation of reactive oxygen species. EMBO J 2007;26:4457-4466.

24 Rakoff-Nahoum S, Paglino J, Eslami-Varzaneh F, Edberg S, Medzhitov $R$. Recognition of commensal microflora by Toll-like receptors is required for intestinal homeostasis. Cell 2004;118:229-241.

25 Fortemaison N, Miot F, Dumont JE, Dremier S. Regulation of $\mathrm{H}_{2} \mathrm{O}_{2}$ generation in thyroid cells does not involve Rac1 activation. Eur $J$ Endocrinol 2005;152:127-133.

26 Eckmann L, Neish AS. NF-кB and mucosal homeostasis. Curr Top Microbiol Immunol 2011;349:145-158.

27 Babbin BA, Jesaitis AJ, Ivanov Al, Kelly D, Laukoetter M, Nava P, Parkos CA, Nusrat A. Formyl peptide receptor-1 activation enhances intestinal epithelial cell restitution through phosphatidylinositol 3-kinase-dependent activation of Rac1 and CDC42. J Immunol 2007;179:8112-8121.

28 Cheng G, Lambeth JD. NOXO1, regulation of lipid binding, localization, and activation of Nox1 by the Phox homology (PX) domain. J Biol Chem 2004;279:4737-4742.

29 Jones RM, Mercante JW, Neish AS. Reactive oxygen production induced by the gut microbiota: pharmacotherapeutic implications. Curr Med Chem 2012;19:1519-1529.

30 Abramov AY, Duchen MR. The role of an astrocytic NADPH oxidase in the neurotoxicity of amyloid $\beta$ peptides. Philos Trans $R$ Soc Lond $B$ Biol Sci 2005;360:2309-2314.

31 Chen XL, Kunsch C. Induction of cytoprotective genes through Nrf2/antioxidant response element pathway: a new therapeutic approach for the treatment of inflammatory diseases. Curr Pharm Des 2004;10:879-891. 